Supporting information for:

\title{
Multiscale Temporal Variations of Atmospheric Mercury Distinguished by the Hilbert-Huang Transform Analysis Reveals Multiple El Niño- Southern Oscillation Links
}

Ly Sy Phu Nguyen ${ }^{1,2,3}$, Kien Trong Nguyen ${ }^{4}$, Stephen M. Griffith ${ }^{1}$, Guey-Rong Sheu ${ }^{1,5, *}$, Ming-Cheng Yen ${ }^{1}$, Shuenn-Chin Chang ${ }^{6}$, Neng-Huei Lin ${ }^{1,5}$

${ }^{1}$ Department of Atmospheric Sciences, National Central University, Jhongli 320, Taiwan

${ }^{2}$ Faculty of Environment, University of Science, Ho Chi Minh City, Vietnam

${ }^{3}$ Vietnam National University, Ho Chi Minh City, Vietnam

${ }^{4}$ Faculty of Electronics Engineering, Posts and Telecommunications Institute of

Technology, Ho Chi Minh City, Vietnam

${ }^{5}$ Center for Environmental Monitoring and Technology, National Central University, Jhongli 320, Taiwan

${ }^{6}$ Environmental Protection Administration, Taipei 10042, Taiwan

*Corresponding Author

Tel.: +886-3-4227151 ext. 65514

E-mail address: grsheu@atm.ncu.edu.tw

Supporting Information Summary

Pages: 17

Figures: 13

Tables: 2 


\section{Contents}

Figure S1: Lulin Atmospheric Background Station (LABS) geographic location and typical East Asia monsoon system in Taiwan................................... 3

Figure S2: Illustration of modulation component and its power spectrum...........4

Figure S3: Illustration of measuring phase difference and delay in the simulated

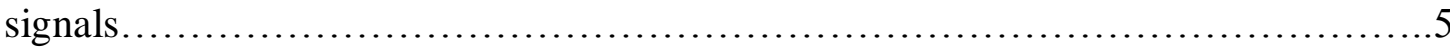

Figure S4: Significance test of the GEM IMFs with the $90 \%$ and $95 \%$ confidence limits................................................................. 6

Figure S5: Hourly GEM observation from LABS and the representative trend (IMF15)

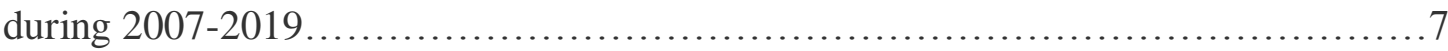

Figure S6: Raw signal and the overall Hilbert time-frequency spectrum of GEM data.8 Figure S7: Marginal HHS plots of IMFs 3 (Diurnal), 8 (Monthly), 10 (Semi-annual),

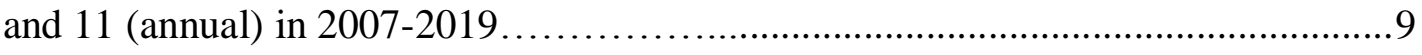

Figure S8: Quantified modulation of diurnal-monthly oscillations.................10

Figure S9: Raw data of SOI and components obtained by EEMD. Accompanying table shows the estimated period and amplitude for each IMF......................... 11

Figure S10: Significance test of the SOI IMFs with the $90 \%$ and $95 \%$ confidence limits

Figure S11: Comparison between GEM and SOI at different IAV modes The y-axis is the normalized data with an arbitrary unit......................................13

Figure S12: Significance test of the CO IMFs with the $90 \%$ and $95 \%$ confidence limits

Figure S13: Comparison between the yearly modes of the GEM and SST (TAO/TRITON).

Table S1: Sensitivity test with variate ensemble members $(50,100,150)$ and a constant added-noise value of 0.3 .

Table S2: Sensitivity test with variate added-noise values of 0.2 to 0.4 standard deviation of the raw signal and a constant ensemble value of 100 . 


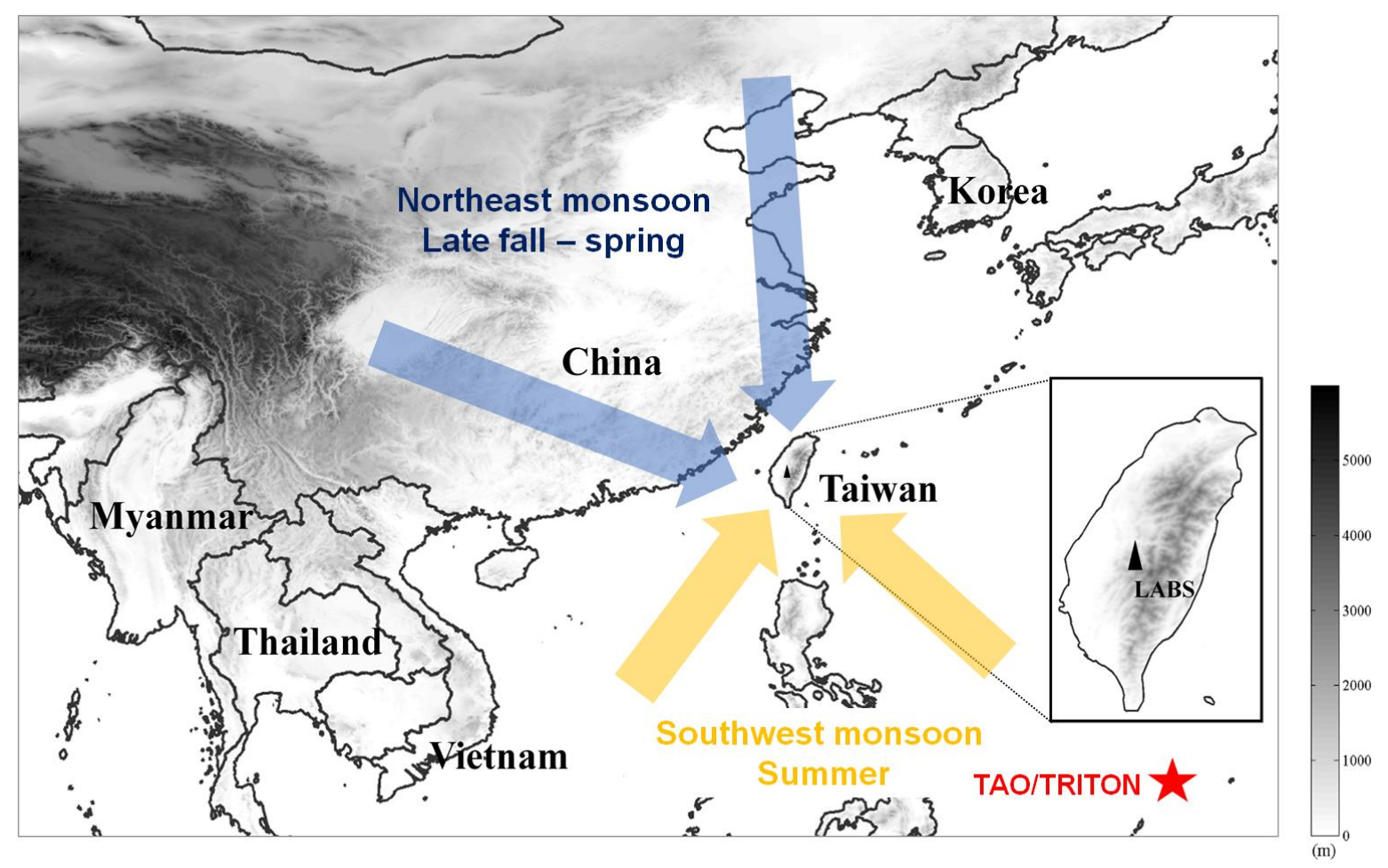

Fig. S1. Lulin Atmospheric Background Station (LABS) geographic location and typical East Asia monsoon system in Taiwan. The red star represents the location of the buoy $\left(8^{\circ} \mathrm{N}\right.$ $137^{\circ} \mathrm{E}$; Tropical Atmospheric Ocean/Triangle Trans-Ocean Buoy Network). 


Fig. S2. Illustration of modulation component and its power spectrum. (a) The signal S was
generated by a sum of sinusoidal signal with frequency of $10 \mathrm{~Hz}$ and white Gaussian noise (N) (b)
The EEMD decomposed signal X into ten IMFs. (c) The envelope of the fifth IMF and its power
spectrum using fast Fourier transform. Here, for demonstration, we only used the fifth IMF, however
this analysis can be applied to other IMFs.




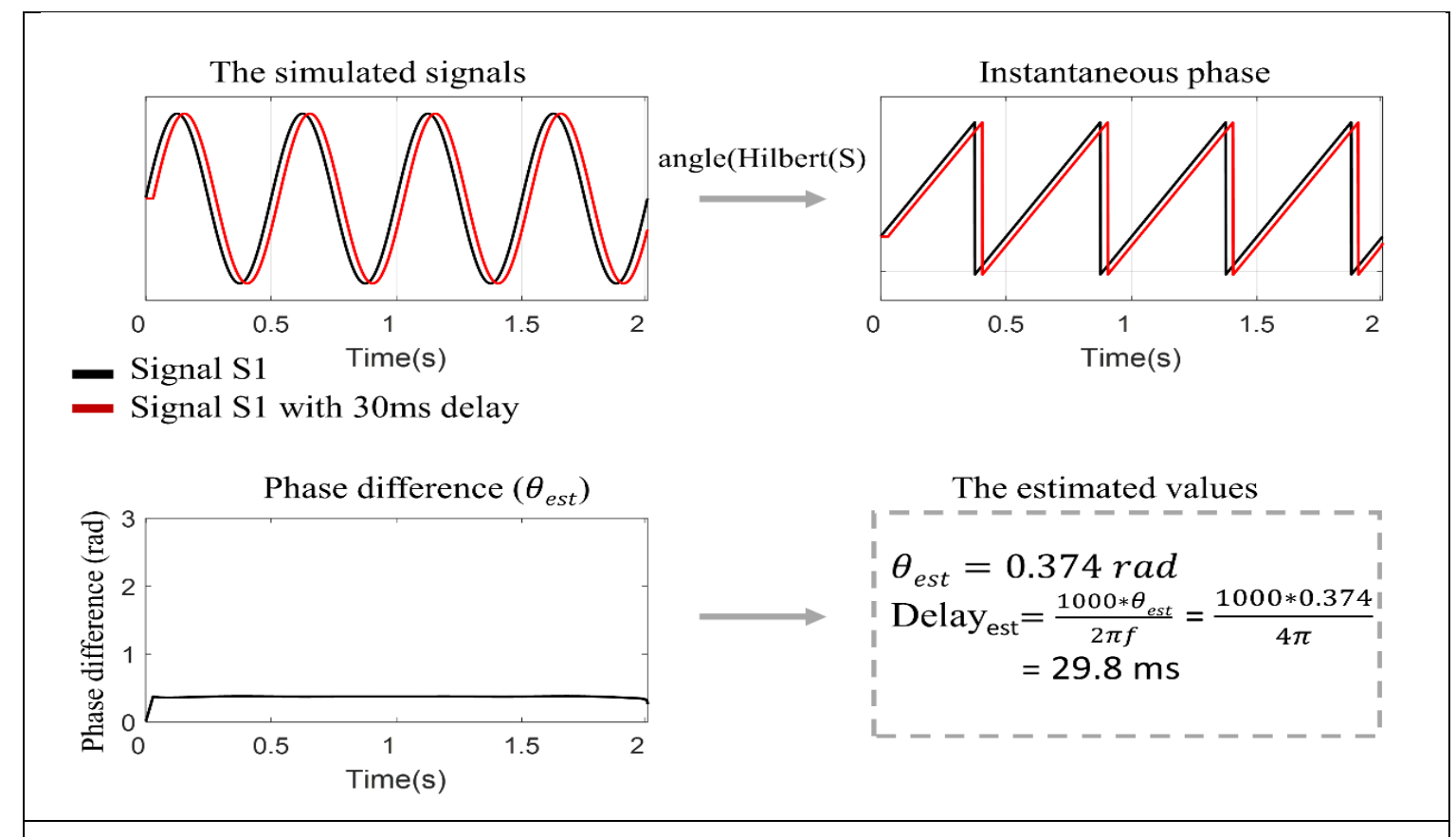

Fig. S3. Illustration of measuring phase difference and delay in the simulated signals. 


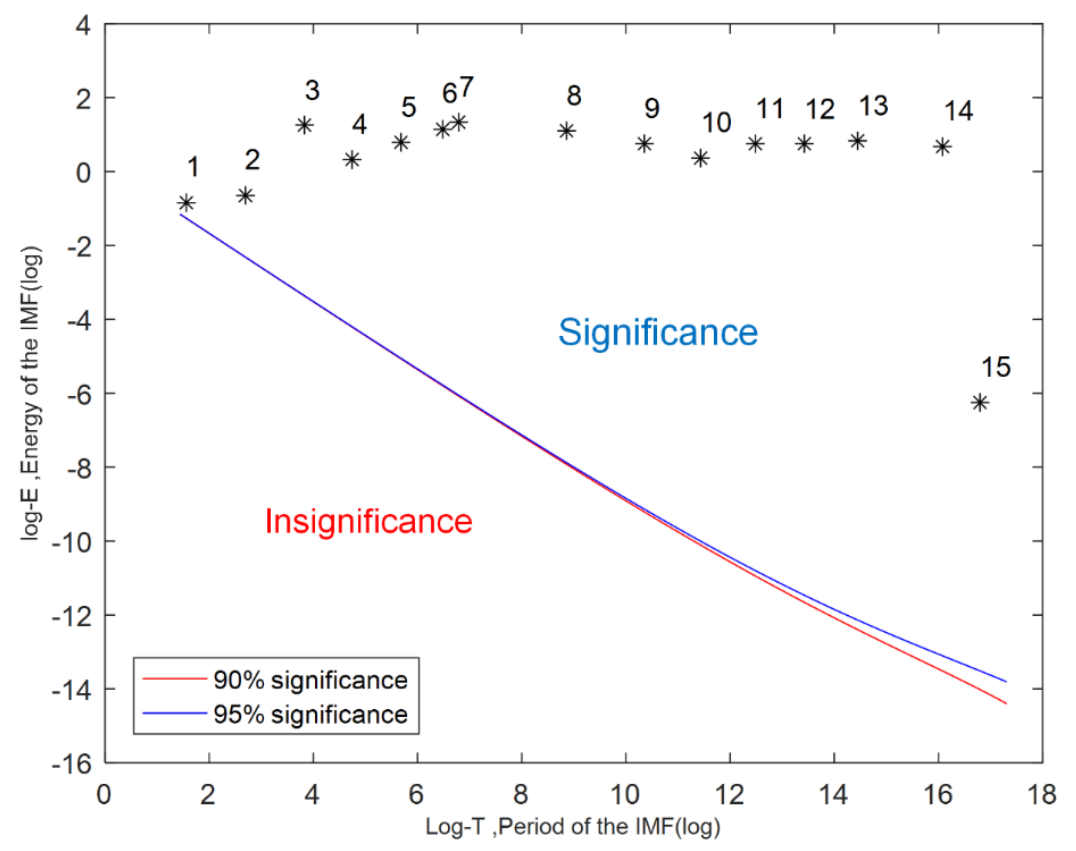

Fig. S4. Significance test of the GEM IMFs with the 90\% (red line) and 95\% (blue line) confidence limits. Each asterisk point below the red (blue) lines indicates that the corresponding IMF of observations significantly differs from the corresponding IMF extracted from white noise at the $90 \%(95 \%)$ confidence levels. 


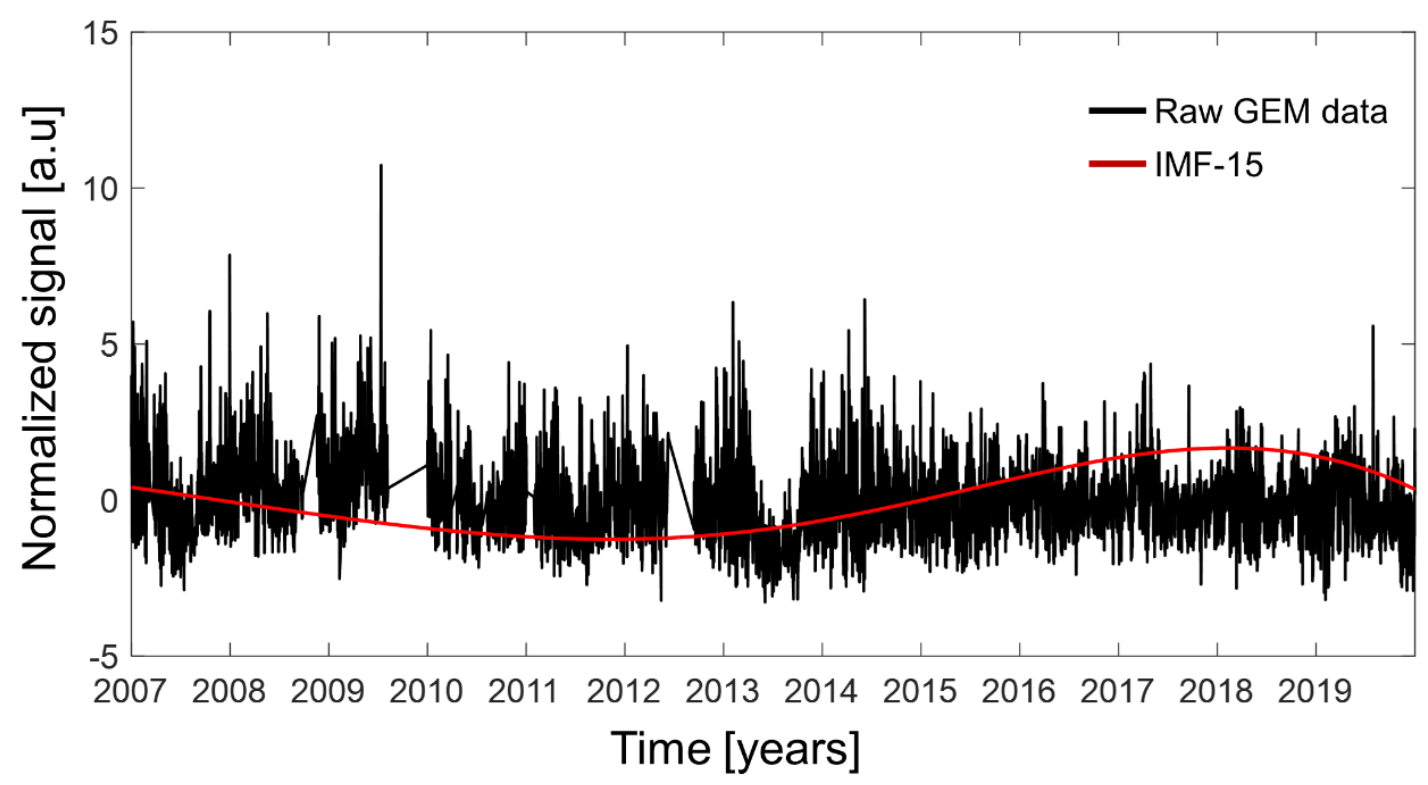

Fig. S5. Hourly GEM observations from LABS (black) and the representative trend (IMF15, red) during 2007-2019. Data has been normalized by subtracting the mean and dividing by the standard deviation. The $\mathrm{x}$-axis represents the time [years]; the $\mathrm{y}$-axis is the normalized data with an arbitrary unit. 


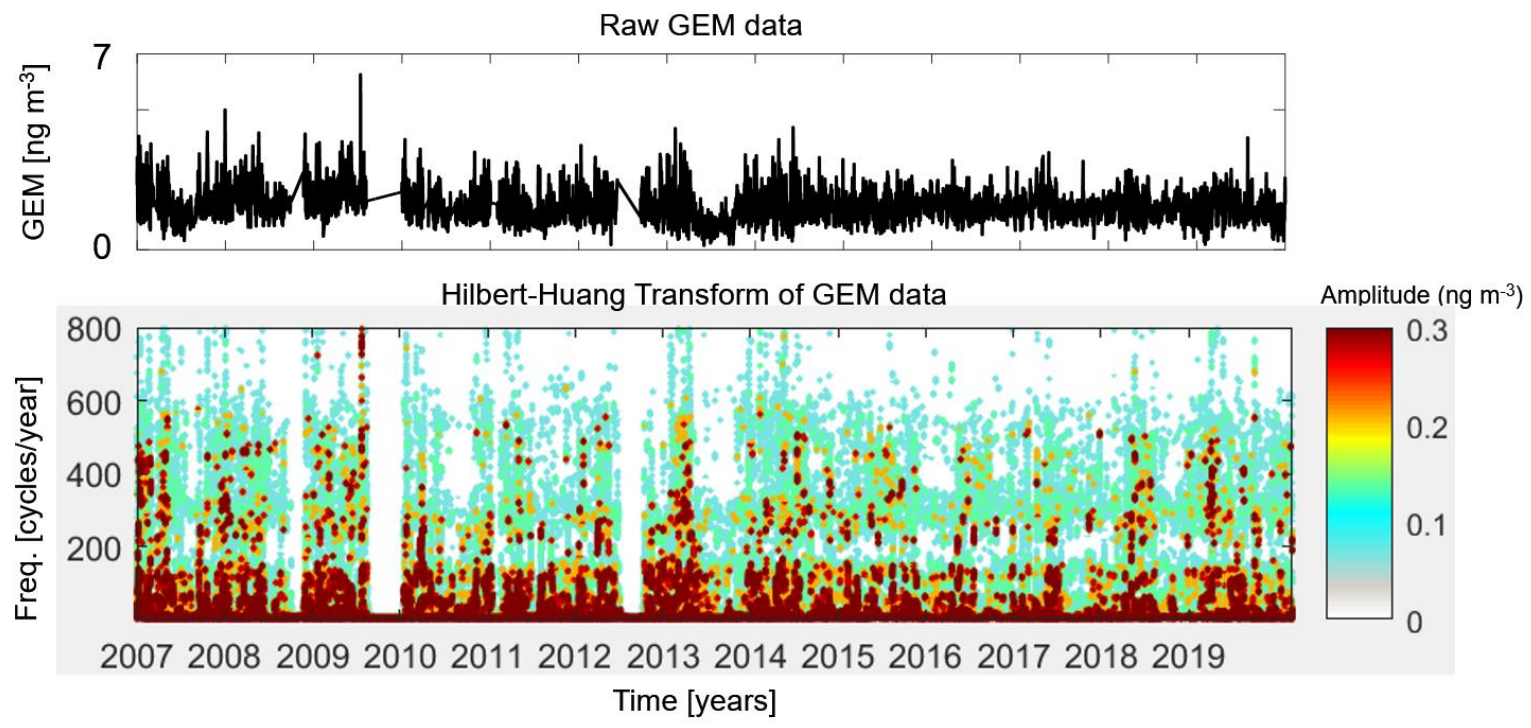

Fig. S6. Raw GEM data and the overall Hilbert time-frequency spectrum of GEM data. Color gradient from strong amplitude (in red) to weak amplitude (in white) highlights the energy activity related to GEM concentrations during the study period. This plot represents 15 overlapping Hilbert Spectra (corresponding to each of the 15 IMFs). 

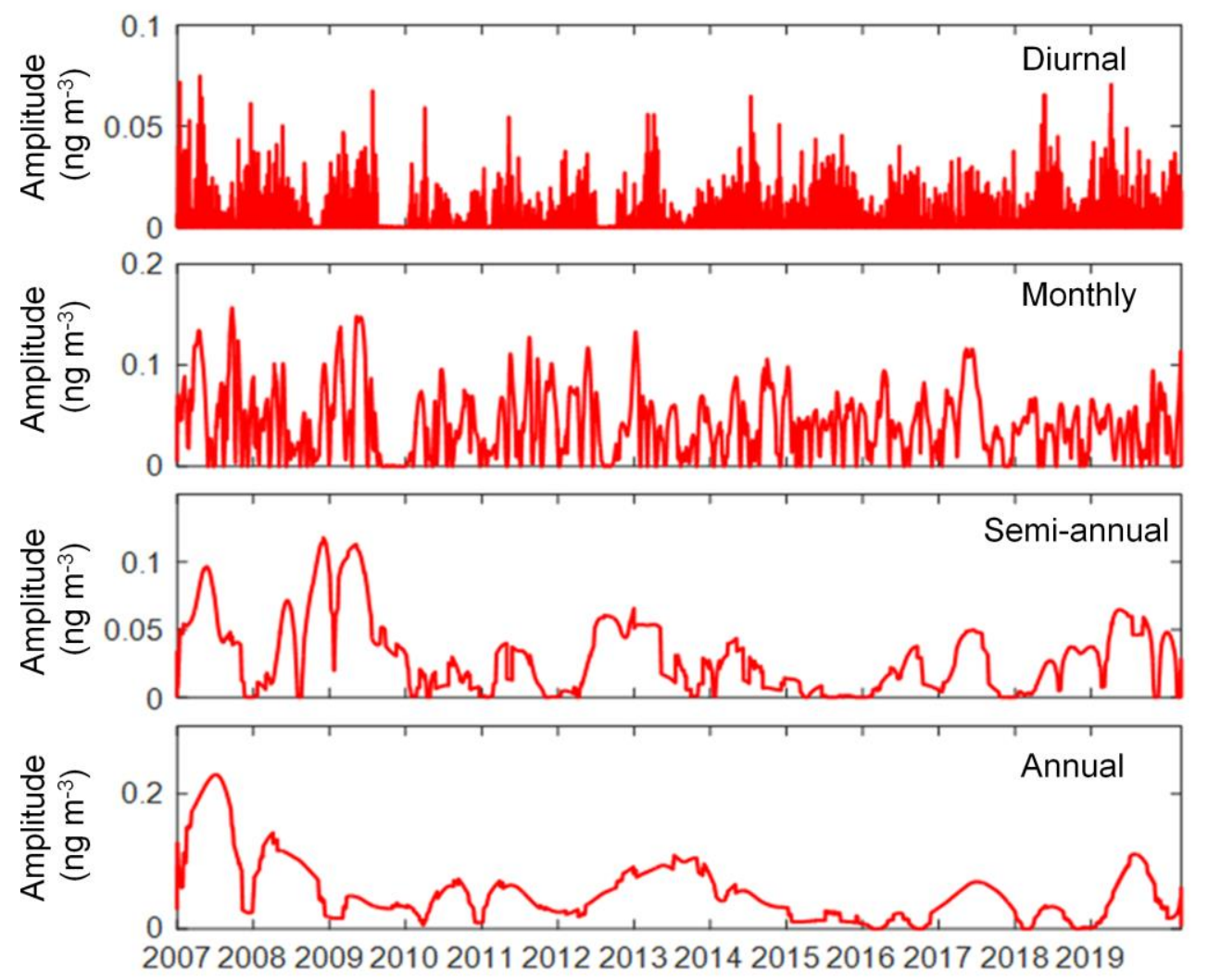

Fig. S7. Marginal HHS plots of IMFs 3 (Diurnal), 8 (Monthly), 10 (Semi-annual), and 11 (annual) in 2007-2019. 

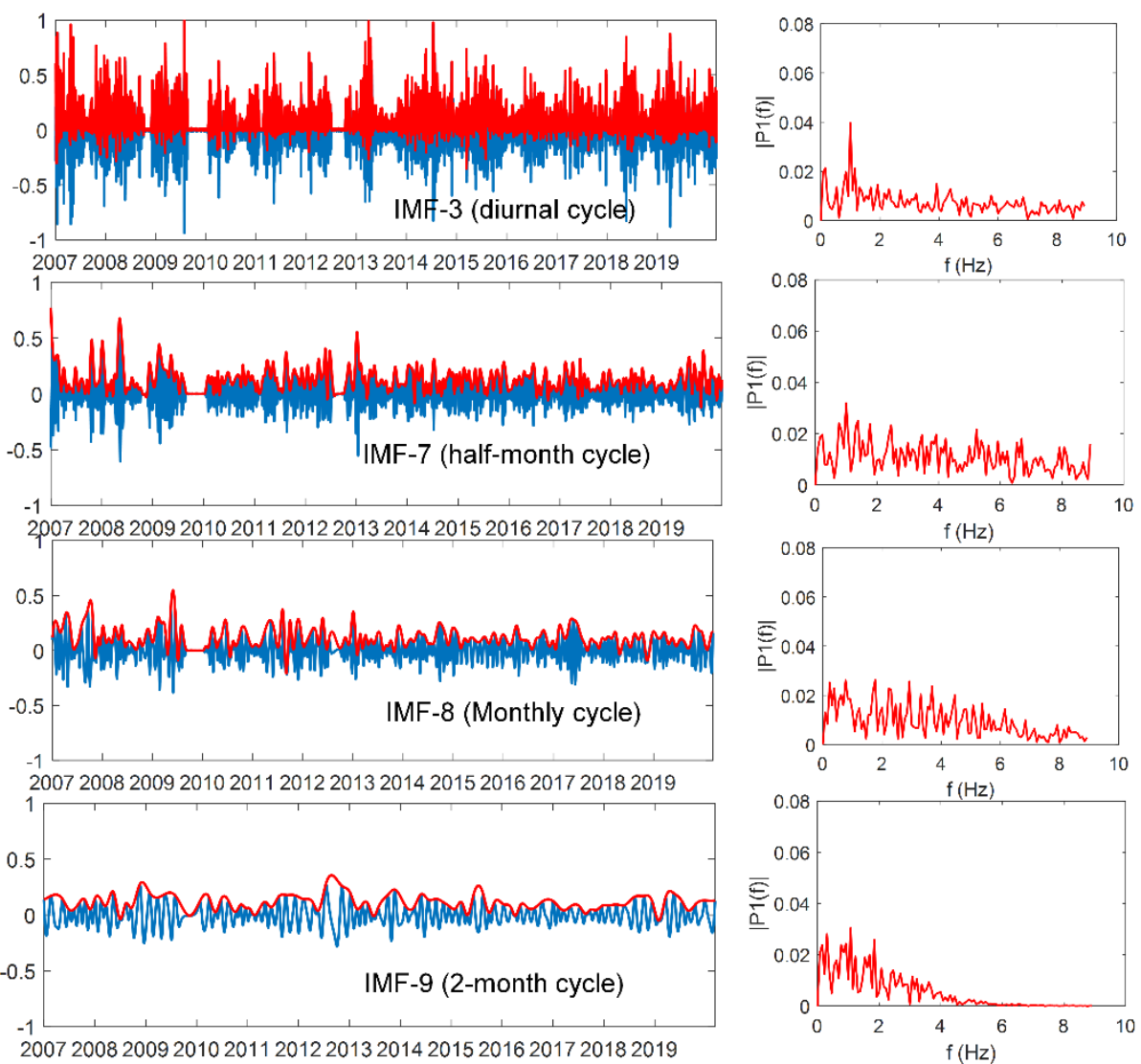

Fig. S8. Quantified modulation of diurnal-monthly oscillations. 


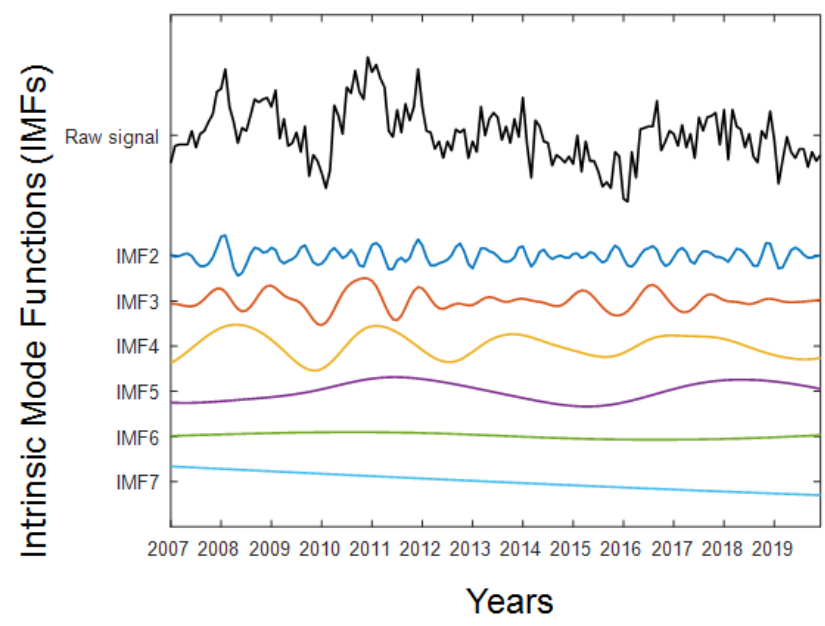

\begin{tabular}{|ccc|}
\hline IMF & $\begin{array}{c}\text { Period } \\
\text { (days) }\end{array}$ & Amplitude \\
\hline IMF\#2 & 279.3 & 0.343 \\
\hline IMF\#3 & 526.5 & 0.544 \\
\hline IMF\#4 & $3-4$ (years) & 0.813 \\
\hline IMF\#5 & $7-8$ (years) & 0.836 \\
\hline IMF\#6 & 13 (years) & 0.249 \\
\hline
\end{tabular}

Fig. S9. Raw data of SOI and components obtained by EEMD. The table shows the estimated period and amplitude for each IMF. 


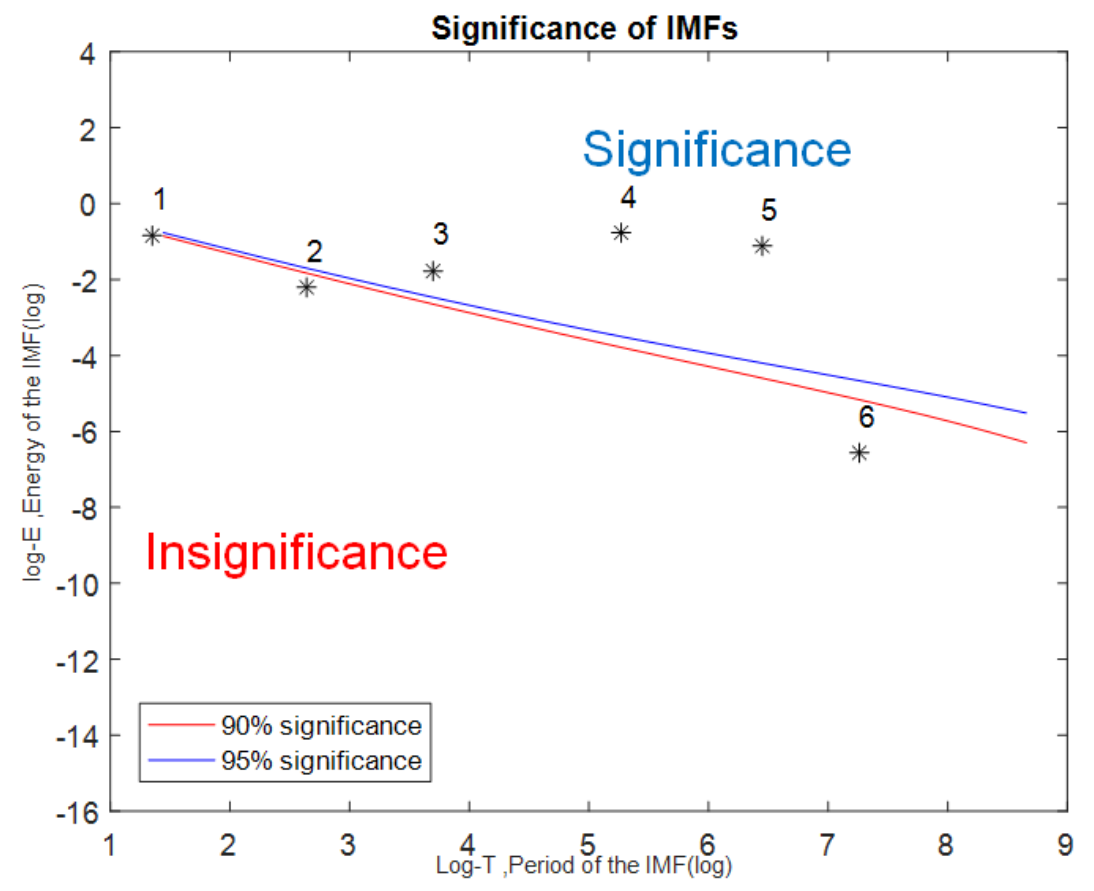

Fig. S10. Significance test of the SOI IMFs with the 90\% (red line) and 95\% (blue line) confidence limits. 


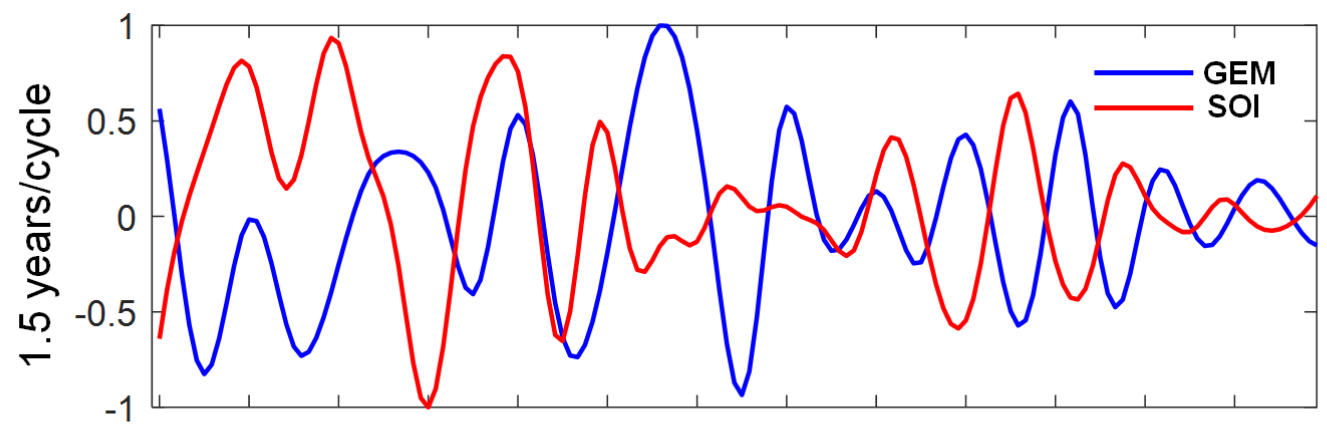

2007200820092010201120122013201420152016201720182019

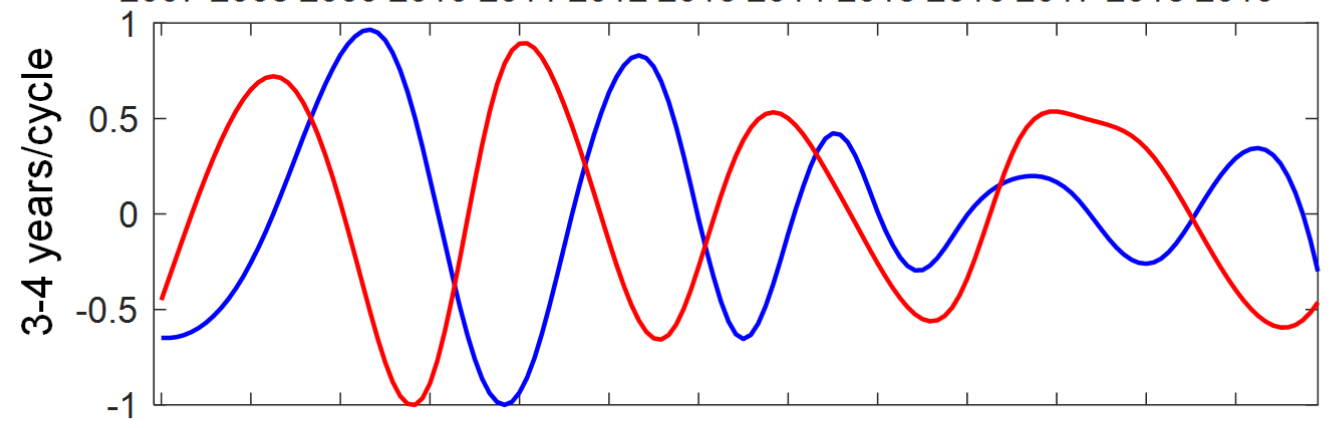

2007200820092010201120122013201420152016201720182019

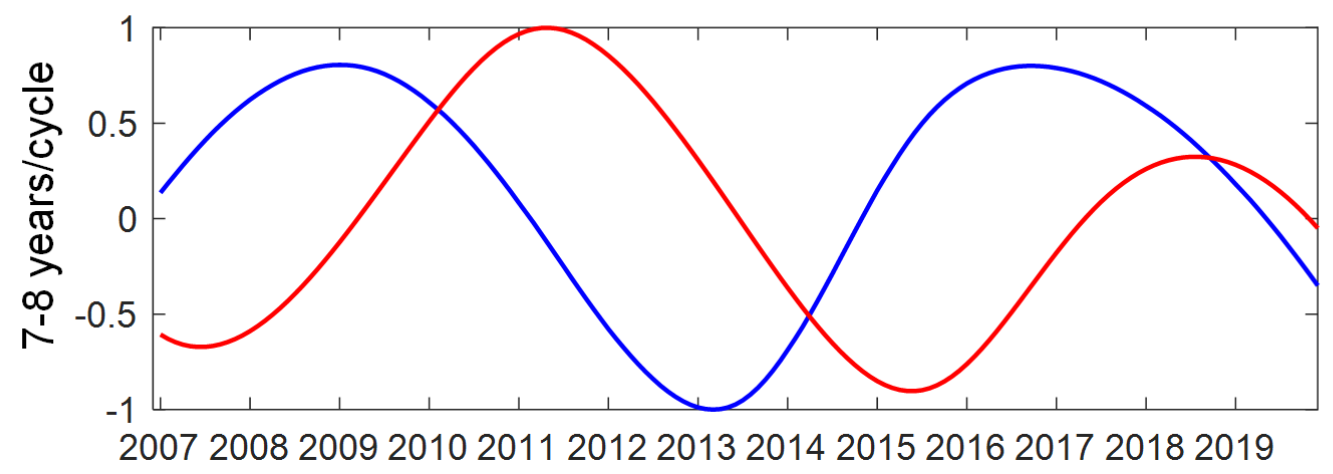

Fig. S11. Comparison between GEM and SOI at different IAV modes. The y-axis is the normalized data with an arbitrary unit. 


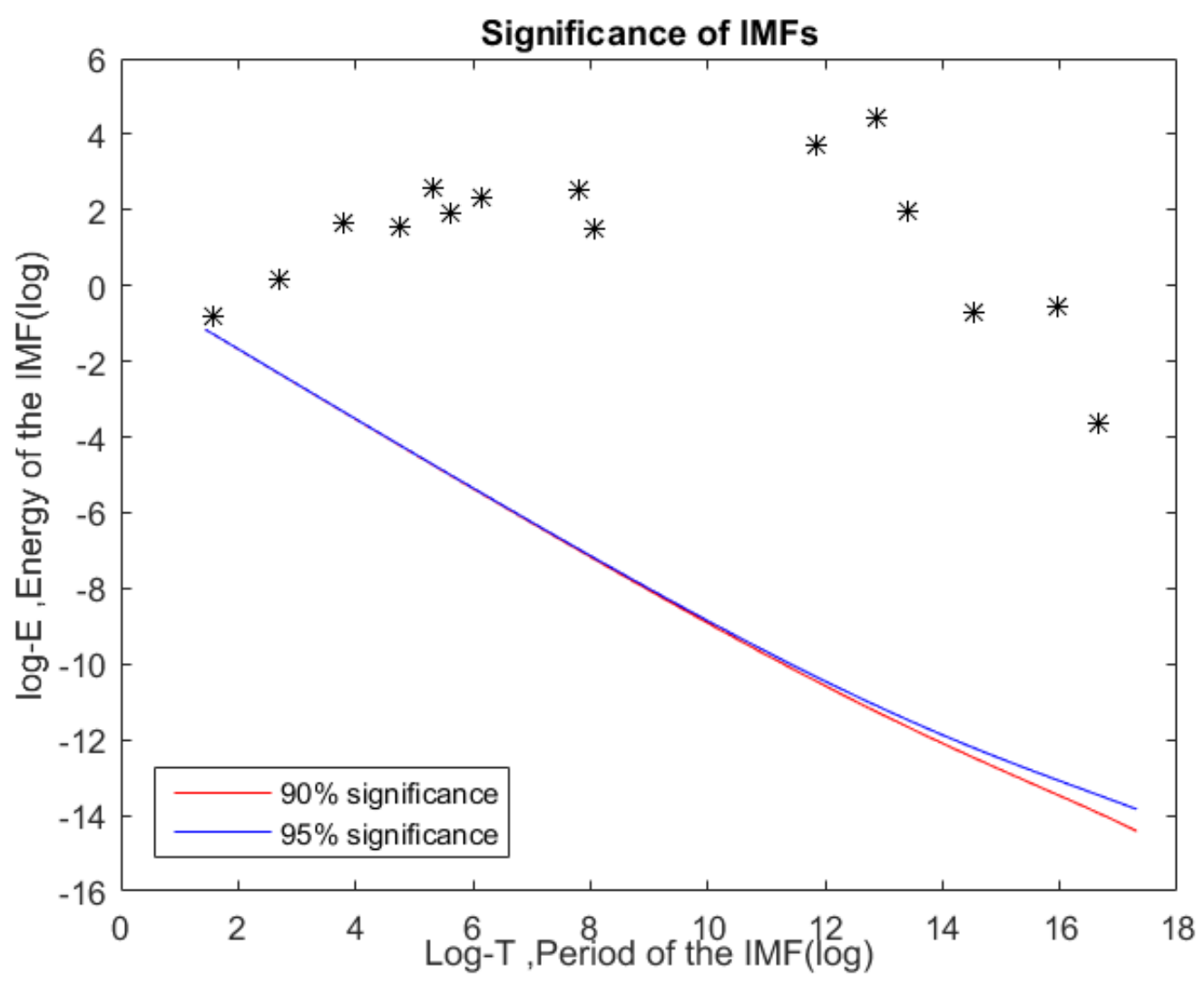

Fig. S12. Significance test of the CO IMFs with the $90 \%$ (red line) and 95\% (blue line) confidence limits. 


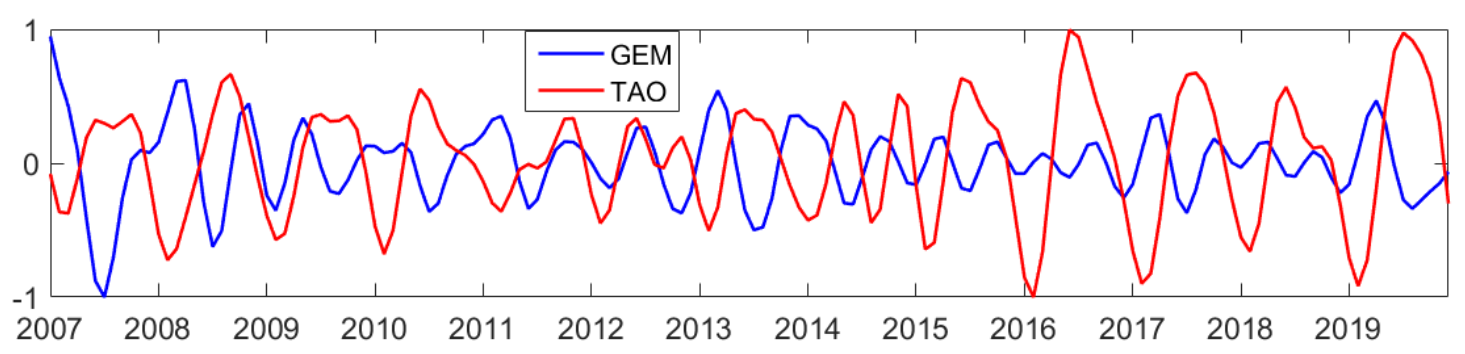

Fig. S13. Comparison between the yearly modes of the GEM and SST (TAO/TRITON). All data sets extend from 2007 to 2019. 
Table S1: Sensitivity test with variate ensemble values $(50,100,150)$ and a constant addednoise value of 0.3 standard deviation of the raw signal. Values reported as mean \pm S.D.

\begin{tabular}{lcc}
\hline IMF & Period (days) & Amplitude \\
\hline IMF 2 & $0.5 \pm 0.0$ & $0.009 \pm 0$ \\
IMF 3 & $1.0 \pm 0.0$ & $0.050 \pm 0$ \\
IMF 4 & $1.0 \pm 0.0$ & $0.027 \pm 0$ \\
IMF 5 & $4.7 \pm 0.0$ & $0.009 \pm 0$ \\
IMF 6 & $9.5 \pm 0.0$ & $0.015 \pm 0$ \\
IMF 7 & $17.5 \pm 0.0$ & $0.024 \pm 0$ \\
IMF 8 & $33.5 \pm 0.0$ & $0.024 \pm 0$ \\
IMF 9 & $62.1 \pm 0.0$ & $0.028 \pm 0$ \\
IMF 10 & $182.0 \pm 0.0$ & $0.037 \pm 0.001$ \\
IMF 11 & $364.1 \pm 0.0$ & $0.049 \pm 0.001$ \\
IMF 12 & $\sim 1.5$ (years) & $0.058 \pm 0.001$ \\
IMF 13 & $3-4$ (years) & $0.065 \pm 0.002$ \\
IMF 14 & $7-8$ (years) & $0.106 \pm 0.004$ \\
IMF 15 & 13 (years) & $0.007 \pm 0.001$ \\
\hline
\end{tabular}


Table S2: Sensitivity test with variate added-noise values of 0.2 to 0.4 standard deviation of the raw signal and a constant ensemble value of 100 . Values reported as mean \pm S.D.

\begin{tabular}{lcc}
\hline IMF & Period (days) & Amplitude \\
\hline IMF 2 & $0.5 \pm 0.0$ & $0.009 \pm 0.001$ \\
IMF 3 & $1.0 \pm 0.0$ & $0.050 \pm 0.005$ \\
IMF 4 & $1.0 \pm 0.0$ & $0.027 \pm 0.005$ \\
IMF 5 & $4.5 \pm 0.4$ & $0.009 \pm 0.001$ \\
IMF 6 & $9.5 \pm 0.0$ & $0.015 \pm 0.001$ \\
IMF 7 & $17.5 \pm 0.0$ & $0.024 \pm 0.002$ \\
IMF 8 & $32.1 \pm 7.1$ & $0.024 \pm 0.001$ \\
IMF 9 & $62.1 \pm 0.0$ & $0.028 \pm 0.001$ \\
IMF 10 & $182.0 \pm 0.0$ & $0.039 \pm 0.007$ \\
IMF 11 & $364.1 \pm 0.0$ & $0.05 \pm 0.006$ \\
IMF 12 & 1.5 (years) & $0.057 \pm 0.002$ \\
IMF 13 & $3-4$ (years) & $0.067 \pm 0.002$ \\
IMF 14 & $7-8$ (years) & $0.109 \pm 0.003$ \\
IMF 15 & 13 (years) & $0.011 \pm 0.011$ \\
\hline
\end{tabular}

